\title{
Article \\ Smart Cleaner: A New Autonomous Indoor Disinfection Robot for Combating the COVID-19 Pandemic
}

\author{
Kaicheng Ruan, Zehao Wu (1) and Qingsong Xu * (D) \\ Department of Electromechanical Engineering, Faculty of Science and Technology, University of Macau, \\ Macau 999078, China; mc05482@um.edu.mo (K.R.); yb97489@um.edu.mo (Z.W.) \\ * Correspondence: qsxu@um.edu.mo; Tel.: +853-88224278
}

Citation: Ruan, K.; Wu, Z.; Xu, Q. Smart Cleaner: A New Autonomous Indoor Disinfection Robot for Combating the COVID-19 Pandemic. Robotics 2021, 10, 87. https:// doi.org/10.3390/robotics10030087

Academic Editor: Marco Ceccarelli

Received: 8 June 2021

Accepted: 5 July 2021

Published: 12 July 2021

Publisher's Note: MDPI stays neutral with regard to jurisdictional claims in published maps and institutional affiliations.

Copyright: (c) 2021 by the authors. Licensee MDPI, Basel, Switzerland. This article is an open access article distributed under the terms and conditions of the Creative Commons Attribution (CC BY) license (https:/ / creativecommons.org/licenses/by/ $4.0 /)$.

\begin{abstract}
The COVID-19 pandemic imposes an increasing demand for service robots as a substitute for humans to conduct various types of work in contaminated areas. Such work includes logistics, patient care, and disinfection, which can reduce the risk of human exposure to the highly contagious and deadly virus. This paper presents the design and development of Smart Cleaner, which is a new cost-effective autonomous indoor disinfection robot. It integrates a wheeled mobile robot platform and a hydrogen peroxide atomization device for automated disinfection operation in the complex indoor environment. Through the system integration of various hardware components and software programming, a prototype of the disinfection robot has been fabricated for experimental investigation. A simulation study of the drymist hydrogen peroxide disinfection model was carried out to understand the diffusion of disinfectant in a room environment. The effectiveness of the developed robot was verified in practical scenarios, such as hospital, hotel, office, and laboratory. The effect of disinfection was validated by a qualified third-party testing agency. Results demonstrate the high efficiency of the developed disinfection robot dedicated to autonomous indoor disinfection work.
\end{abstract}

Keywords: autonomous robot; service robot; mobile robot; indoor disinfection; SLAM; automation

\section{Introduction}

Since late 2019, the outbreak of novel coronavirus disease (COVID-19) has become a worldwide pandemic, which brings huge pressure to the public healthcare system worldwide. By 4 March 2021, a total of 114,428,211 confirmed cases and 2,543,755 deaths with 348,281 daily increases in new cases were reported by World Health Organization [1]. With no wonder cure and universal vaccine coverage in sight, due to supply constraints and virus variants, one of the key measures to control the spread of COVID-19 and other contagious diseases is to prevent normal people from getting infected, for example, reducing unnecessary interpersonal communication (also known as social distancing), terminal disinfection, and isolation of infection are effective actions. Such approaches have been accepted by most countries worldwide and have shown the desired effect in restricting the escalation of both infection and asymptomatic infection cases.

From a technical point of view, the deployment of robots is a part of the preventative measure to overcome the induced difficulties and boost the efficiency of combating the COVID-19 pandemic. During the past year, various robots have been employed to conduct a series of key tasks and vital functions via different methods [2]. Robots have been classified in the literature as one of the ways of overcoming the challenges of the pandemic [3-5]. It has been found that, overall, robotic systems are overall apt solutions for dealing with many problems caused by COVID-19. These include diagnosis, screening, disinfection, surgery, tele-healthcare, logistics, manufacturing, and broader interpersonal problems unique to the lockdowns of the pandemic [6]. In addition, the robots can be sorted based on different application scenarios [7], such as hospitals, airports, transportation tools, hotels, restaurants, and communities. Autonomous robotic systems have their own potential and advantages in the campaign against COVID-19. The reason lies in that the robots 
are immune to pathogens. Moreover, they can easily access places which humans have difficulty reaching, although several technical problems and challenges remain to be solved by the whole of academia and industry.

For example, robots have been deployed in the field of diagnosis [8,9]. They can reduce the risk of people being exposed to the deadly virus. Remote control can reduce the tiredness of doctors, as the real working environment is usually harsh due to the limitation of disease control, like lack of cooling and ventilation. In the logistics field, a robot can distribute daily supplies to people in lockdown areas [10]. In addition, a smaller robot has been reported for delivering patient's medicines and foods in indoor environments, such as hospital and quarantine hotel room [11].

In this paper, we mainly focus on indoor disinfection, as the COVID-19 virus can survive on the inorganic surface for up to several days or even weeks, depending on what kind of material it was attached to. This may cause a healthy person to get infected in addition to human-to-human proximity contact transmission. Currently, most of the indoor disinfection in the contaminated areas, like hospitals and quarantine hotels, were performed by human workers using chemical disinfectants. This undoubtedly increases the chance of a healthy human becoming exposed to the highly contagious virus, particularly when personal protective equipment were not properly worn or lacked. Moreover, the consistency of each disinfection cannot be guaranteed. Alternatively, autonomous or remote-controlled disinfection robots could lead to safe, fast, and effective disinfection [3].

Currently, air and surface disinfection work is carried out using various disinfectants, such as peroxyacetic acid, chlorine dioxide, hydrogen peroxide, etc. Among them, hydrogen peroxide is a clean disinfectant, which can be catalytically converted to oxygen and water vapor, leaving residue-free air and surface in the environment [12]. Hence, it has been widely used to replace conventional disinfectants such as formaldehyde and ozone. For indoor space disinfection, hydrogen peroxide liquid of low concentrations (e.g., below $10 \%$ ) can be sprayed directly. However, such an approach will cause wastage of hydrogen peroxide. To utilize the hydrogen peroxide more efficiently, there are two main approaches, i.e., vaporized hydrogen peroxide and drymist hydrogen peroxide (DHP) technologies [13]. As compared to the former, the latter method produces smaller particle size $(0.5-5 \mu \mathrm{m})$ with easier maintenance and lower cost for disinfection and sterilization of spaces [14]. Hence, DHP technology is preferable for large-scale application in indoor space without pollution.

Another disinfection method that has been adopted uses ultraviolet-C (UVC) lights [15]. Generally, UVC lamps have a specific working distance, depending on what kind of reactor is used. After leaving its maximum radiation zone, the effect of disinfection drops dramatically due to energy loss. As a result, some rear areas of a complex environment cannot be irradiated due to the path restriction of the robot. Meanwhile, the light generated from the UVC lamp cannot penetrate opaque surfaces. Thus, there will definitely be a dead zone of disinfection if one surface is behind another. This would be a potential risk because highly contagious respiratory droplets from a patient can be diffused to the air and surface even at a long distance. Hence, in this work, the disinfection is implemented by adopting the drymist hydrogen peroxide approach.

In particular, the drymist hydrogen peroxide is an Environmental Protection Agencyregistered sporicidal sterilant with in vitro and in situ efficacy against nosocomial pathogens. It has demonstrated feasibility in outbreak remediation and has been linked with reductions in acquisition of healthcare-associated pathogens and infection. More importantly, it can be diffused into a space for more uniform and continuous disinfection, without being blocked by other surfaces. After reaching the air and surface, the hydrogen peroxide acts as an oxidizing agent and produces reactive oxygen species to attack the components of the pathogens, such as DNA, RNA, and protein shell [16]. Although there are no direct data showing the effect of disinfecting COVID-19 using hydrogen peroxide in the literature, previous research confirms its effectiveness in eliminating various pathogens ranging from 
H1N1 influenza virus [17] to transmissible gastroenteritis coronavirus, which is quite like that of COVID-19 [18].

The main contribution of this paper is the design and development of a novel costeffective autonomous disinfection robot system for extensive disinfection applications. To the knowledge of the authors, no similar work on disinfection robot development with DHP has been reported in the literature. The following parts of the paper are organized as follows. Section 2 presents the design process of the autonomous disinfection robot. Section 3 details the simulation study of drymist hydrogen peroxide disinfection model. The robot navigation and path planning is implemented in Section 4. With the developed disinfection robot, Section 5 outlines the disinfection testing and experimental results of the developed disinfection robot. Section 6 concludes this paper.

\section{Design of the Autonomous Disinfection Robot}

The design process of the autonomous disinfection robot is presented in the following.

\subsection{Mechanism Design}

A CAD model of the designed disinfection robot is shown in Figure 1. The robot structure consists of two parts, i.e., the autonomous moving platform and the disinfection module. The autonomous moving platform is used to carry the disinfection module for navigation in the designated environment based on simultaneous localization and mapping (SLAM) [19]. The disinfection module is realized by an ultrasonic atomizer, which is able to convert hydrogen peroxide liquid into micrometer-sized drymist for efficient space disinfection.

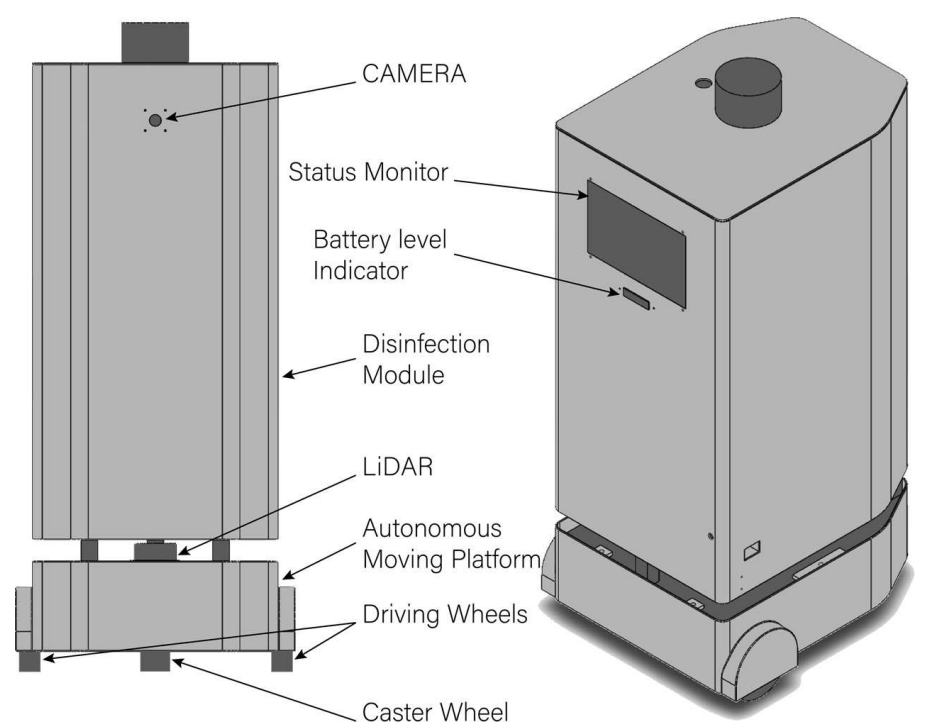

Figure 1. CAD model of the designed autonomous disinfection robot.

The autonomous moving platform is a mobile robot with two differential driving wheels. It is designed to achieve high mobility ability (e.g., moving forward, moving backward, and turning itself around) in an indoor environment for disinfection application. In addition, a caster wheel is used to keep the balance of the platform on the ground. For moving in a limited indoor environment, the planar diameter of the robot platform is designed to be as compact as possible within a diameter of $500 \mathrm{~mm}$.

For carrying the disinfection equipment and other accessories (such as sensors and control board), a supporting frame is designed for the mobile robot. To realize full-space disinfection and sterilization, a drymist hydrogen peroxide sterilizer is adopted and integrated into the upper supporting frame of the mobile robot. To maintain a compact design, the height of the entire robot is kept within $1200 \mathrm{~mm}$. 


\subsection{Sensing and Control Implementation}

For realizing obstacle avoidance, localization, motion planning, and navigation, it is necessary for the robot to obtain environment information by perception [20]. Mobile robot perception can be conducted by detecting recognizable features of the environment using different types of sensors [21]. Robot vision and laser scanners are useful sensors for environment perception [22]. In this way, the meaningful elements (e.g., objects and scenes) in the environment can be detected to for complete knowledge of the environment for carrying out the movement safely and reliably. Based on this, by conducting initial path planning, robot navigation can be accomplished whereby unexpected static or dynamic obstacles are avoided while tracking the motion trajectory $[23,24]$.

Figure 2 illustrates the connection diagram of the power supply and control hardware of the robot system. In addition to carry the disinfection module, the autonomous moving platform also contains several supporting components, including control boards, lidar, and a battery capable of supplying multiple voltages (e.g., 5, 12, and $24 \mathrm{~V}$ ) for providing a suitable power supply for the disinfection module. The ultrasonic atomizer disinfection module is used to atomize the hydrogen peroxide disinfectant at a rate of $1500 \mathrm{~mL} / \mathrm{h}$ in the indoor environment.

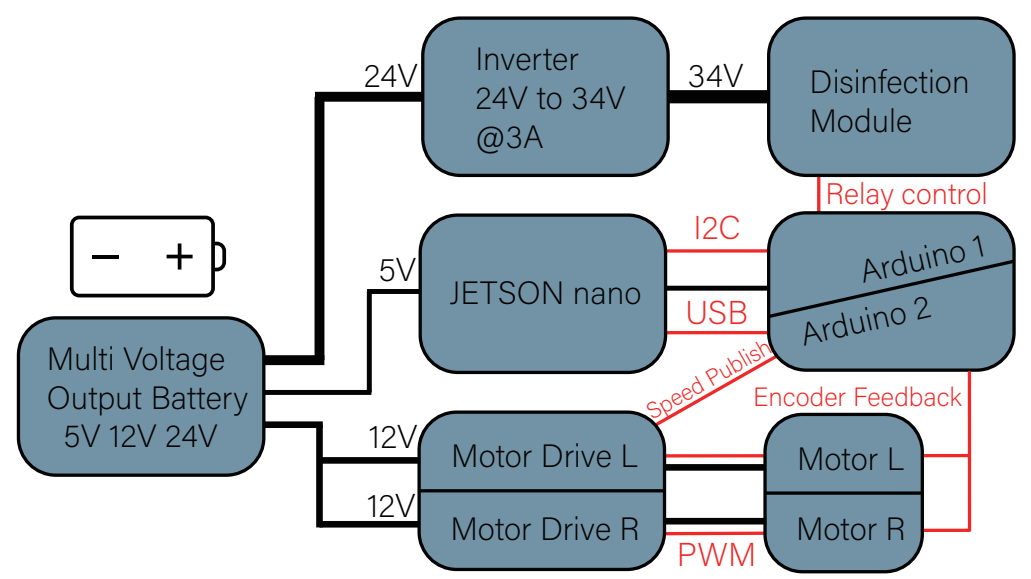

Figure 2. Block diagram of the connection between control hardware and power supply.

The core of the control system is the upper-level controller (or master controller). In this work, the embedded control board (model: JETSON Nano, from Nvidia Corp., Santa Clara, CA, USA) was adopted and programmed on the basis of Robot Operating System (ROS) to handle all of the input and output channels. Typical input includes the data of surrounding distance from the lidar (model: RPlidar A2, from Shanghai Slamtec Co., Ltd., Shanghai, China), which are acquired to detect obstacles in the nearby environment for avoiding possible collisions. In combination with the odometry data from the encoder of the driving wheels (the resolution of wheel encoders is $2550\left(17^{*} 150\right)$, the basic pulse is $17 \mathrm{ppr}$, and the gear ratio is 1:150), it facilitates map establishment of the designated area for use in navigation.

In addition, the upper-level controller also receives control command from the operator or controller itself, and then publishes it to the lower-level controller for further processing. The lower-lever controller is realized by adopting two microcontroller boards (model: Arduino Mega 2560, from Arduino LLC). Typical output includes the robot movement speed, image captured by built-in camera, and so on. These data are then transmitted to the remote controller (e.g., personal computer) with a monitoring screen.

\subsection{System Integration and Control Modes of the Robot}

To develop the entire robot prototype, the aforementioned hardware components including mobile robot platform, supporting frame, hydrogen peroxide sterilizer, sensors, and control board are integrated together. A schematic view of the robotic platform's 
software architecture is shown in Figure 3. For replacing human workers to perform automated disinfection work, the autonomous robot system has been programmed under a Linux operating system to realize the automation process. The disinfection robot is controlled by three modes, including two auto modes and one manual mode.

In the manual control mode, the operator uses a remote stick controller or keyboard to control the movement and switch of the robot. This facilitates rapid disinfection depending on operator's independent judgment without requiring the map of the environment. It is also used to manually navigate the robot for scanning the environment to establish the environment map for auto-navigation.

After the map is generated, the auto mode can be used to simplify the disinfection procedure. The first auto mode is the route recording mode, which is a method upgraded from the previous one by manually setting several key points. The robot itself can plan a suitable route to disinfect the designated area repeatedly, and it tries to avoid obstacles during this process. The second auto mode is the route planning mode, i.e., a remote planning method that draws the key points and target forward angle of the robot based on the map to disinfect the designated area repeatedly. It also tries to avoid obstacles during this process, which can be used in the environment not suitable for humans to stay.

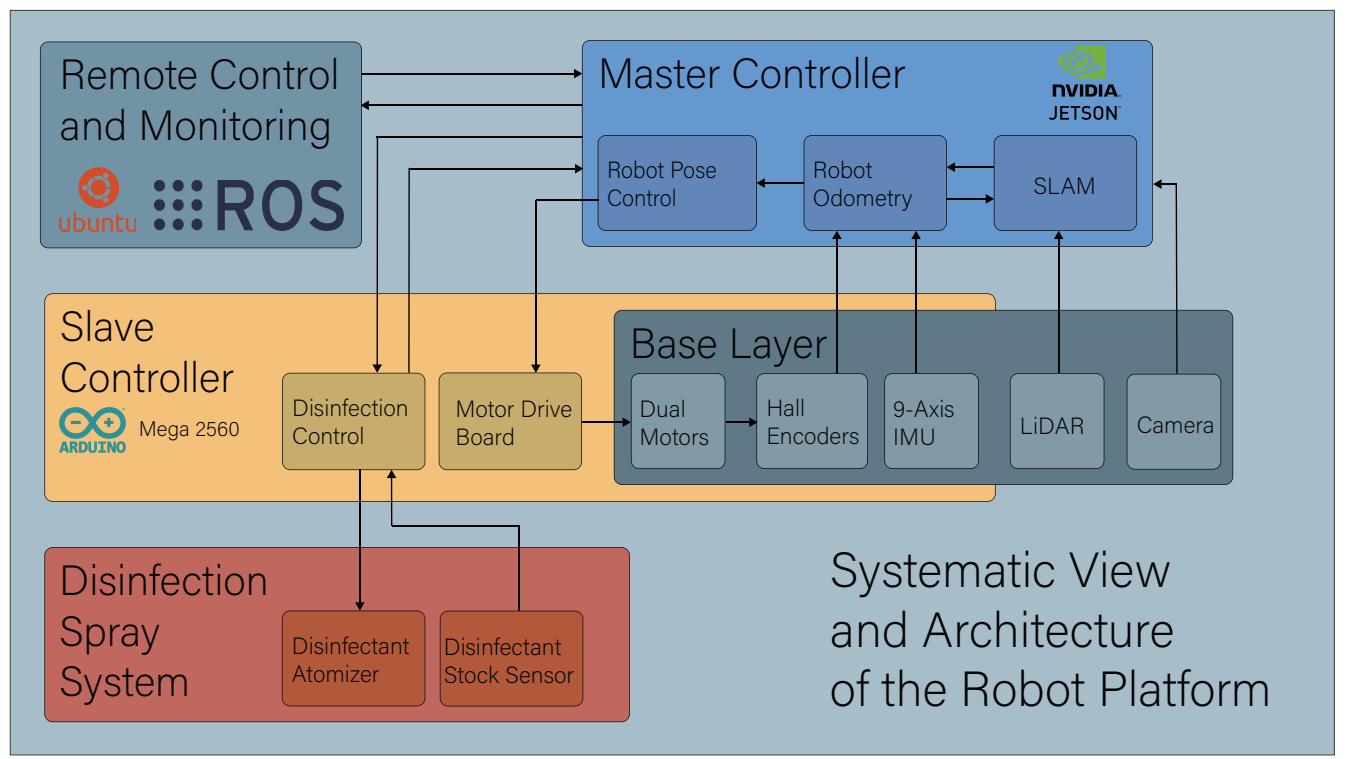

Figure 3. Schematic view of the software architecture for the developed disinfection robot. The arrows indicate the publishing/receiving of the data, command stream, and the relation between each part.

\section{Simulation Study of Drymist Hydrogen Peroxide Disinfection Model}

While there are plenty of studies about robots with UVC disinfection, investigations of robots with DHP are rare in the literature. With the help of the rapid development of computer aided simulation method and computational fluid dynamics (CFD), a flow field model for DHP diffusing in a limited space is simulated in this section. Through this model, the diffusion method of the DHP is discussed, which is helpful for the research and application of the space disinfection robot using DHP.

The existing studies concerning DHP are mainly focused on the disinfection effect on various pathogens ranging from the sterilization of microorganisms to the elimination of viruses. In comparison, little research has been conducted about the diffusion and distribution of the DHP disinfection in real scenarios, especially when the indoor environment is complex, with furniture and ventilation involved. The previous work [25] has simulated the situation of DHP disinfection in a hospital operating room, with DHP mixing with the air coming from the air-conditioning flow at the top of the room. However, in the case of a disinfection robot, the DHP inlet is usually at the middle height of the room. This is determined by the height of the inlet port of the disinfection robot. In addition, the inlet 
port is moving along with the robot rather than being stationary, and the concentration is also different. Moreover, the inlet port of the air-conditioning pipe is larger than that in the disinfection robot. The robot also involves different structures of diffusing head for delivering DHP into the space.

\subsection{Concentration Selection for Disinfection}

The concentration of DHP for deactivating the COVID-19 virus has not been clearly defined yet. Even so, several types of research can be referred to which set the required concentration in simulations. For example, Rudnick et al. found that after $2.5 \mathrm{~min}$, the exposure to $10 \mathrm{ppm}$ DHP resulted in $99 \%$ inactivation of H1N1 influenza viruses [17]. The cleaning and disinfection of environmental surfaces in the context of COVID-19 from WHO shows that $0.5 \%$ of hydrogen peroxide can achieve more than $3 \log 10$ reductions of human coronavirus for environmental surface cleaning [26]. Another report from CDC also indicated that $0.5 \%$ accelerated hydrogen peroxide demonstrated bactericidal and viricidal activity in $1 \mathrm{~min}$, and mycobactericidal and fungicidal activity in $5 \mathrm{~min}$ [27], while the disinfectant user guide published by the General Office of the National Health Commission (NHC) of China suggests the use of 3\% hydrogen peroxide for indoor air disinfection [28]. In this paper, we adopt $1 \%$ concentration as the indicator for the completion of surface disinfection, and $3 \%$ for the completion of indoor air disinfection.

\subsection{Setup of Test Environment}

In the simulation study, the disinfection space is assigned as an L-shaped room. The shape and dimensions of the room are illustrated in Figure 4. The size of the robot is $0.3 \mathrm{~m} \times 0.2 \mathrm{~m} \times 0.55 \mathrm{~m}$ with an inlet pipe of $0.05 \mathrm{~m}$ diameter and $0.65 \mathrm{~m}$ length. The robot is located in the centerline of the room, being $0.2 \mathrm{~m}$ away from the inner wall. The DHP enters the disinfection space through the inlet port mounted at the center of the robot. The size of the ventilation fan is $0.3 \mathrm{~m} \times 0.3 \mathrm{~m}$, which is located at the corner ceiling of the space and is closed during the disinfection. Whereas the possibility of causing passive air inlet should be taken into consideration, another condition that needs to be considered is air leakage from the clearance (size: $0.9 \mathrm{~m} \times 0.02 \mathrm{~m}$ ) at the bottom of the room door. All of the dimensions were measured using a laser rangefinder (model: LM80, from Uni-Trend Technology (China) Co., Ltd., Dongguan, China). The initial temperature and humidity of the indoor environment were $27^{\circ} \mathrm{C}(300 \mathrm{~K})$ and $90.6 \%$, respectively, as measured by a windmeter (model: UT-333 BT, from Uni-Trend Technology (Dongguan, China) Co., Ltd.).

\subsection{Model Detail and Boundary Condition}

During the simulation of the disinfection case study, the influence of the buoyancy airflow effect was ignored to simplify the calculation of the internal fluid flow field. In addition, as the fluid velocity is relatively slow $(0.1$ to $3 \mathrm{~m} / \mathrm{s})$, the air can be defined as incompressible matter. The energy equation and species transport were enabled to solve the diffusion problem. It was found that the standard $k-\varepsilon$ model in Fluent was enough to solve the diffusion of the DHP in the aforementioned space. The SIMPLE scheme was chosen as the solving method. For setting the boundary condition, we used UT-363 windmeter (from Uni-Trend Technology (China) Co., Ltd.) to measure the average wind speed in the operating space and inlet port. The speed of the DHP inlet was set as $3 \mathrm{~m} / \mathrm{s}$, and the speed of passive air inlet was $0.1 \mathrm{~m} / \mathrm{s}$. The clearance of the door was set as the pressure outlet. In addition, the concentration of DHP in the inlet was assigned as 7\%, which is the same as the disinfectant used in practice. 


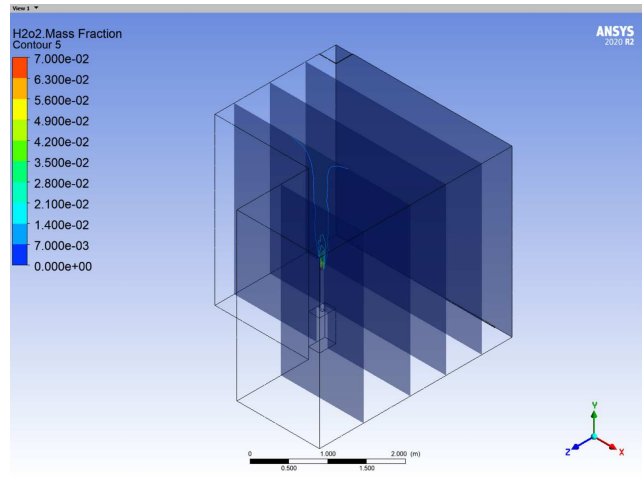

(a)

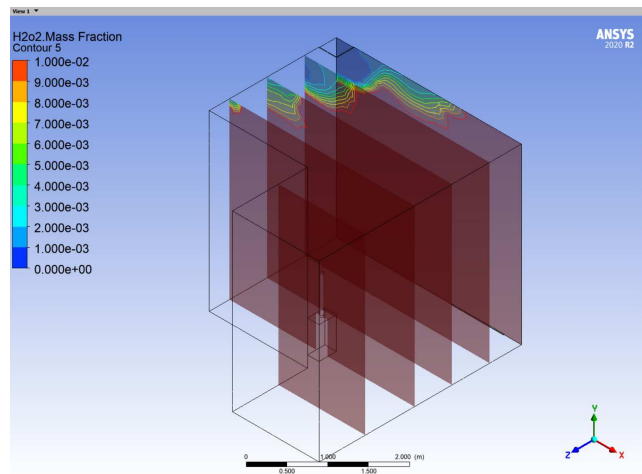

(c)



(b)

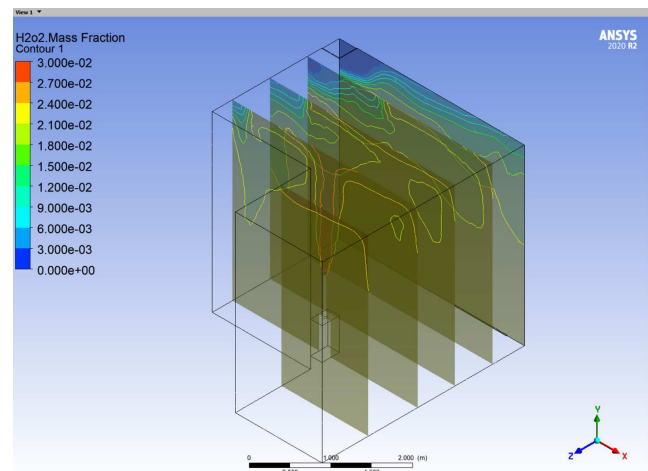

(d)

Figure 4. Simulation results of contour plots for disinfectant diffusion in a room the time instants of (a) $120 \mathrm{~s}$, (b) $850 \mathrm{~s}$, (c) $1200 \mathrm{~s}$, and (d) $3000 \mathrm{~s}$.

\subsection{Simulation Results}

For better illustration, five planes were inserted into the disinfection space evenly, displaying the contours of the $\mathrm{H}_{2} \mathrm{O}_{2}$ mass fraction of the plane surfaces. The image was saved once per 5 timesteps, with one timestep set as $1 \mathrm{~s}$. After delivering DHP for $120 \mathrm{~s}$, the simulation results of contour plots are shown in Figure 4a. After delivery for 850 and $1200 \mathrm{~s}$, the mass fraction contours are as shown in Figure $4 \mathrm{~b}, \mathrm{c}$, respectively. It is seen that as the delivery time continues, the DHP keeps spread through space. At around $850 \mathrm{~s}$, the surfaces of the disinfection space reach the concentration of $1 \%$, which satisfies the requirement of surface disinfection. It takes another $350 \mathrm{~s}$ for DHP to diffuse across space, except for the space near the passive air inlet. The total simulation time was set as $3000 \mathrm{~s}$. This was still not enough for the entire concentration of the DHP to reach 3\%, which is required by the NHC of China for air disinfection. The highest spread of DHP is concentrated near the inlet in the space. A longer disinfection time or higher concentration of $\mathrm{H}_{2} \mathrm{O}_{2}$ disinfectant are expected to achieve effective disinfection.

When the inlet speed of the DHP is set as $3 \mathrm{~m} / \mathrm{s}$ and the concentration is $7 \%$, about $850 \mathrm{~s}$ is required for the DHP to diffuse around the surface. In addition, it takes $1200 \mathrm{~s}$ to cross a room without ideal sealing. For further optimization, the diffusing head can be redesigned to allow DHP flow in multiple directions, which can realize the distribution of DHP more evenly. As the robot is not stationary, a route-based diffusion can be realized to improve the disinfection performance. In future work, experimental study will be implemented by using bio indicator or other methods to validate the CFD model and optimize related parameters based on the acquired data. 


\section{Navigation and Path Planning Implementation}

Auto-navigation in an unfamiliar environment is required to endow the robot with the capability of generating its action plan for tracking [29]. In particular, the localization, path planning, and navigation strategy need to be implemented online [30]. Mobile robot navigation can be solved using three different approaches, i.e., geometric navigation, topological navigation, and semantic navigation. As a well-known geometric navigation way, SLAM [31,32] has been popularly adopted to generate metric maps, which are used to guide the robot for moving through the map with metric path planners. For instance, SLAM has been used together with rapidly-exploring random tree (RTT) planning and Monte Carlo localization to navigate a mobile robot in indoor environments [33].

The following ROS packages (open source) were used in this work: rplidar_ros [34] is a driver that provides basic device handling between ROS and lidar; gmapping [35] is a ROS wrapper for OpenSlam's Gmapping; move_base [36] provides an implementation of action, e.g., given a goal in the world frame, it will attempt to reach it with a mobile base; openni2 [37] is a driver which allows basic device handling between ROS and RGB-D camera; amcl [38] is a probabilistic localization system for a robot moving in 2D space; and rosserial [39] is a protocol for wrapping standard ROS serialized messages and multiplexing multiple topics and services over a character device, such as a serial port or network socket.

\subsection{SLAM Implementation}

In this work, for guiding the robot to move autonomously for disinfection, the SLAM algorithm is adopted to build the environment map. The main function of SLAM is to analyze the input data and to determine the pose of the robot and build a map. For a mobile robot, sensors such as wheel speed encoders, IMU, or RGB-D camera can be applied to provide the odometry. A 2D/3D lidar or RGB-D camera is then adopted to build the map.

In the application of disinfection, the wheel speed encoder is designated to calculate the odometry because the floor of these scenes are generally composed of porcelain tile, which is not easy to skid. Moreover, a high-precision wheel speed encoder is used to get accurate input data which can better finish the mobile disinfection work. In addition, 2D-lidar is also adopted for mapping. It considers the shapes of most objects in this application scene, such as beds, cabinets, and chairs. Most of their shapes are regular. Therefore, low-cost 2D-lidar is sufficient for identifying the objects accurately, which avoids the deployment of expensive 3D-lidar.

The most common algorithms in 2D-lidar SLAM include Gmapping, Hector, Cartographer, etc. Gmapping algorithm is used here due to its portability and robustness. It is very suitable for the establishment of a small scene map. As the disinfection scenes, such as hospital waiting room or hotel room, are usually on a small scale, its mapping effect is complete and accurate enough. As shown in Figure 5, the generated grid map of a small cafeteria in hotel has a trapezoid shape. The wall thickness of the generated map is thin and can clearly and unambiguously reflect reality, which is sufficient for the disinfection work. 


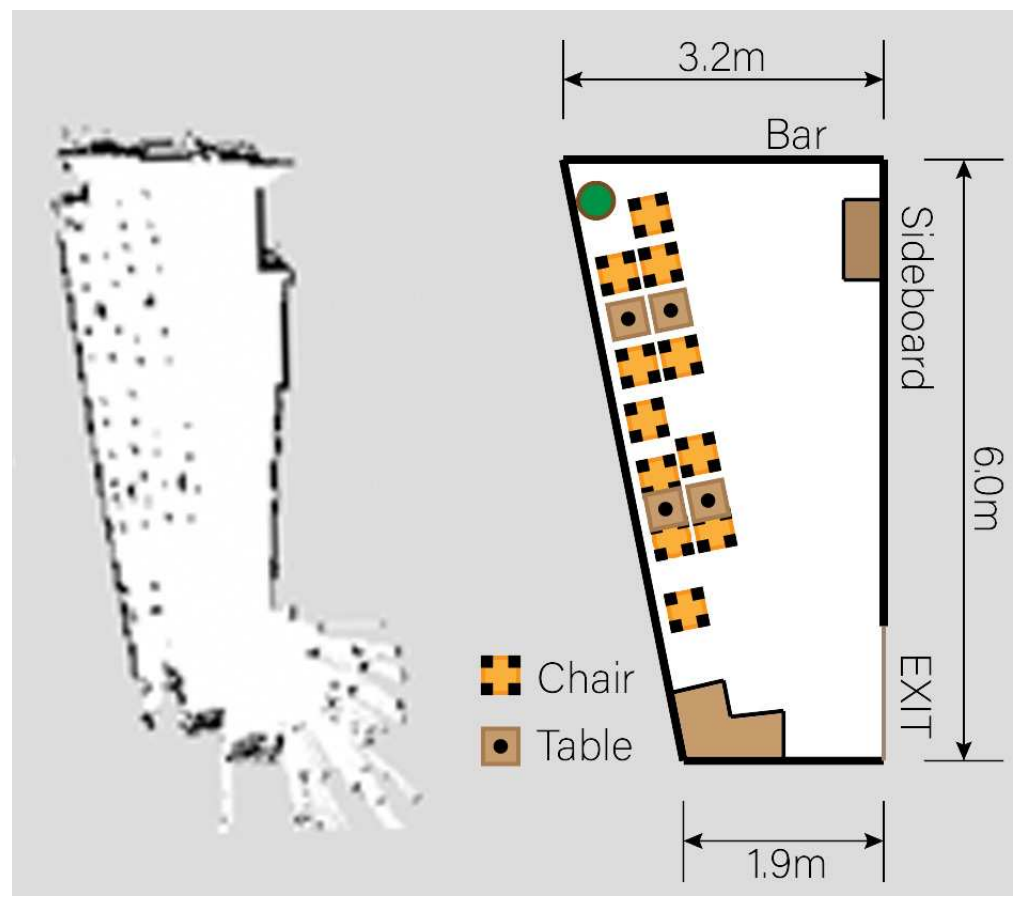

Figure 5. The map generated by the SLAM (left) and actual floor plan of the hotel cafeteria (right). The black point indicates the structure of furniture in lidar scan layer.

\subsection{Gmapping Method}

Gmapping is a SLAM algorithm based on 2D-lidar. It adopts Rao-Blackwellized particle filter (RBPF) to build a two-dimensional grid map. Specifically, it decomposes the SLAM problem into two parts, localization and mapping, through the conditional joint distribution [40]. Gmapping uses loop closure and odometry data. Unlike other SLAM methods such as Hector-SLAM, the Gmapping algorithm was chosen here owing to its ability of supporting odometry data provided by the robot.

\subsection{Path Planning}

After building the map, it is necessary to solve the single-point path planning problem to enable the robot to perform multi-point disinfection safely at a specified location in the generated 2D grid map. In this work, a global path planner and a local path planner are used to jointly plan a single-point path. Among them, the global path planner can quickly calculate the path of the robot to the target point in the grid map. However, there may be some obstacles (such as people) suddenly appearing on the pre-planned path, which are not recorded in the original map. In this scenario, the local path planner will perform partial secondary path planning to avoid obstacles. In this way, the robot can finally reach its destination safely.

\subsubsection{Global Planning}

For the application scenes in this work, the $\mathrm{A}^{*}$ algorithm is adopted as the global path planning method. The $\mathrm{A}^{*}$ algorithm is a commonly used graph traversal method. It finds the node with the smallest value of the priority function in the priority queue as the next traversal node continuously, and keeps traversing until reaching the target goal [41]. Therefore, it can plan the best path with the least cost, which is also the main reason of adopting this algorithm.

\subsubsection{Local Planner}

The algorithm of dynamic window approach (DWA) is used for the local planner. The DWA algorithm mainly samples multiple sets of velocities in the velocity space. It simulates the trajectories of these velocities within a certain time. Then, these trajectories 
are scored by an evaluation function, and the optimal path with the highest score is selected [42].

Actually, the DWA is adopted here because its calculation time is short and the local path can be updated in real time. In addition, there are no dead angle obstacles or local optimization in the disinfection scenes. Hence, the DWA can complete the task of avoiding obstacles well.

\subsubsection{Overall Planning}

By combining the global and local path planners, the disinfection robot can avoid obstacles by planning a safe path to the goal autonomously.

For illustration, Figure 6 shows the real test result of the path planning of the disinfection robot in a laboratory. Firstly, Figure 6a shows the comprehensive path (the green line segment) generated after the target point is given. However, there is a sudden obstacle (such as a person) appearing on its original planned path, which can be seen in Figure 6b. Thus, the local planner will replan and change the path to avoid the obstacle. This is the conclusion of a complete single-point path planning event.

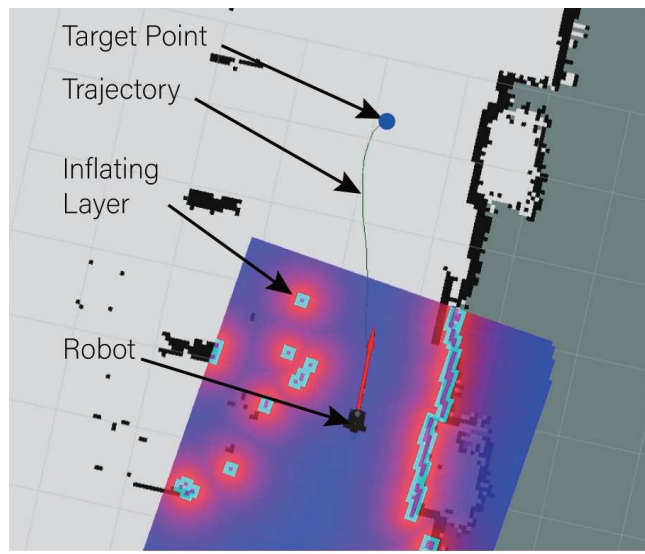

(a)

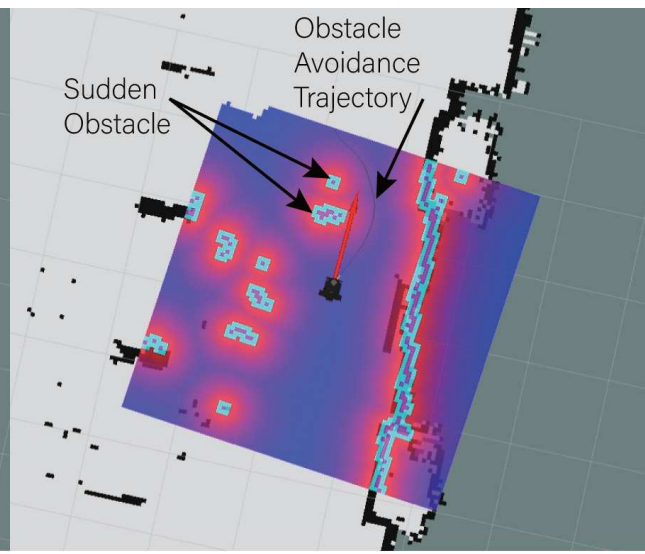

(b)

Figure 6. Path planning test in a laboratory: (a) a trajectory generated through $\mathrm{A}^{*}$ and DWA after target point; (b) a replanned trajectory is generated when a sudden obstacle appears on its originally planned path.

In the normal disinfection scene, we can preset the positions of multiple target points and set the program to execute continually. Thus, the disinfection cycle between multiple points is completed.

\subsection{Accuracy Testing of the Localization}

The prerequisite of high efficiency and effective disinfection method relies on the accurate route of the robot traveling, from which we can calculate the disinfected space area. It also requires the support from highly accurate map of the operating environment. Both of them need stable and accurate localization of the robot.

To evaluate the accuracy of the localization and SLAM, we first need to acquire the actual data of the test environment. The test is conducted in a close space without sudden obstacle interfering, while the table, chair, shelf, and other common furniture are included to simulate the real-world situation as authentically as possible. The layout of the area $(6 \mathrm{~m} \times 12 \mathrm{~m})$ is shown in Figure 7. After finishing the setup and measurement of the test environment, the manual mode of the robot is used to conduct the map establishment. For the evaluation of localization accuracy, we also adopt the manual mode to control the robot for traveling a preset route. The result is compared with the one from the trajectory generated by the SLAM algorithm without consideration of robot pose and posture. This also serves as the calibration basis for the later comparison of different modes. 

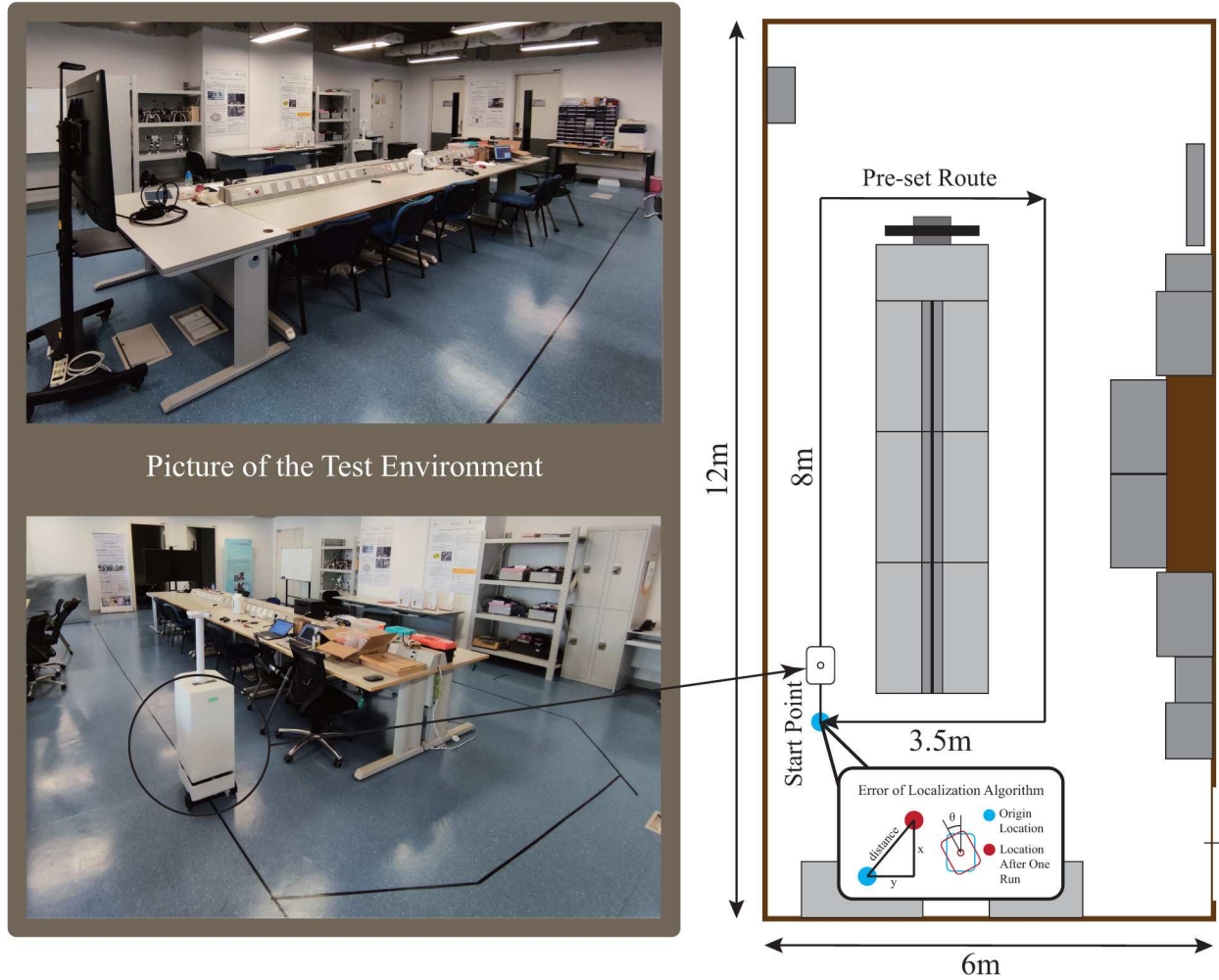

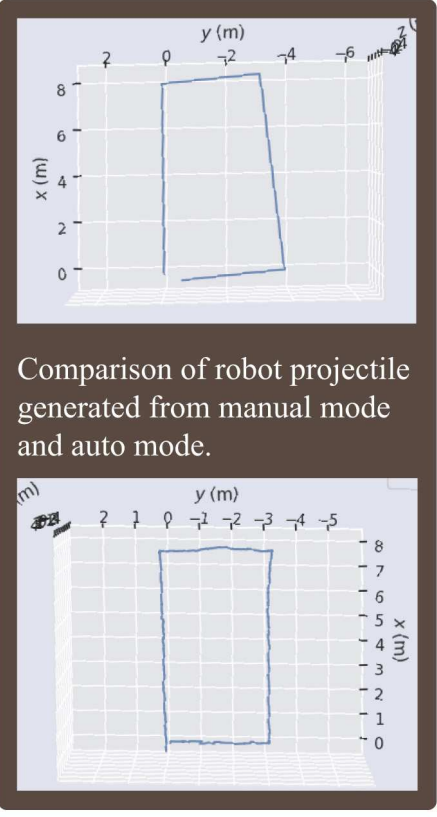

\section{Test Environment and the comparison of the diferent mode}

Figure 7. Photo (left) and planar diagram (middle) of the test environment in a laboratory, with the trajectory tracking results (right) obtained by different robot control modes.

From the comparison result, it is found that the trajectory tracking error generated from the manual mode is relatively large and the trajectory is not closed after the robot returns to the start/finish point because it only obtains the data from the odometer of wheels. After applying the SLAM algorithm, the trajectory generated from the auto mode fits the actual preset route more accurately.

In addition, another test was conducted to quantify the localization accuracy based on this close-ring route. As the start point is the same as the end point in practice, the deviation of the robot's start and end positions after finishing one cycle is considered as the localization error in the test. As the robot structure is rigid, the relative location between the lidar and outer shell is fixed. Thus, we adopted a marker on the ground to verify whether the lidar returned to its original location. The measurement has an uncertainty of $\mathrm{mm}$ range. By calculating the standard deviation of these errors in multiple runs, we can get the quantified localization accuracy. A schematic diagram of localization error is shown in Figure 7, and the test results are tabulated in Table 1.

First, we adopted the Shapiro-Wilk test (W-test) [43] to verify the data normality. It was observed that the significance level $(p)$ value (0.012) of error data y was smaller than 0.05 , which indicates that the error y does not follow normal distribution, while other parameters (with $p>0.05$ ) can be considered as normally distributed. For further analysis, we adopted the position error (rather than individual errors $x$ and $y$ ) and angle error $\theta$ as two evaluation criteria (see Figure 7), which follow normal distributions. Considering the small-sample test, we adopted $t$-distribution to quantify the localization errors. Assigning the confidence interval of $95 \%$, the error ranges for the position and angle errors are calculated as $0.056 \mathrm{~m}$ to $0.073 \mathrm{~m}$ and $-3.807^{\circ}$ to $-1.717^{\circ}$, respectively. 
Table 1. Experimental results of localization error testing.

\begin{tabular}{ccccc}
\hline No. & Error $\mathbf{x}(\mathbf{m})$ & Error $\mathbf{y}(\mathbf{m})$ & Position Error $(\mathbf{m})$ & Angle Error $\boldsymbol{\theta}\left({ }^{\circ}\right)$ \\
\hline 1 & 0.063 & -0.031 & 0.07021 & -3.423 \\
\hline 2 & 0.057 & -0.033 & 0.06586 & -2.578 \\
\hline 3 & 0.063 & -0.031 & 0.07021 & -3.247 \\
\hline 4 & 0.046 & -0.030 & 0.05492 & -1.369 \\
\hline 5 & 0.043 & -0.044 & 0.06152 & -3.193 \\
\hline Average & 0.0544 & -0.0338 & 0.06454 & -2.762 \\
\hline Standard Deviation & 0.00942 & 0.00581 & 0.00648 & 0.84146 \\
\hline Test statistic W of W-test & 0.852 & 0.711 & 0.896 & 0.823 \\
\hline Significance p of W-test & 0.201 & 0.012 & 0.389 & 0.123 \\
\hline
\end{tabular}

\section{Disinfection Testing and Experimental Results}

By integrating the aforementioned techniques, an autonomous indoor disinfection robot has been developed for indoor auto-disinfection in this work. The robot has a compact dimension of $37 \mathrm{~cm} \times 33 \mathrm{~cm} \times 87 \mathrm{~cm}$ with the weight of $25 \mathrm{~kg}$. Moreover, it is a cost-effective robot, and its hardware costs only around USD 3000. The developed disinfection robot has been tested in various indoor environments, including a research laboratory, Wu Yee Sun Library at the University of Macau, Kiang Wu Hospital, and Holiday Inn Express Hotel Macau, among others.

For instance, a small cafeteria in Holiday Inn Express Hotel Macau is shown in Figure 8. The floor plan of the disinfection environment is displayed in Figure 5. To evaluate the disinfection effect, due to the lack of a COVID-19 virus sample, bacteria were used as the effect indicator of disinfection rather than the virus. Actually, bacteria have a more stable biological structure than viruses. This indicates that if this kind of disinfection method can eliminate bacteria, then the more fragile viruses will also be removed.

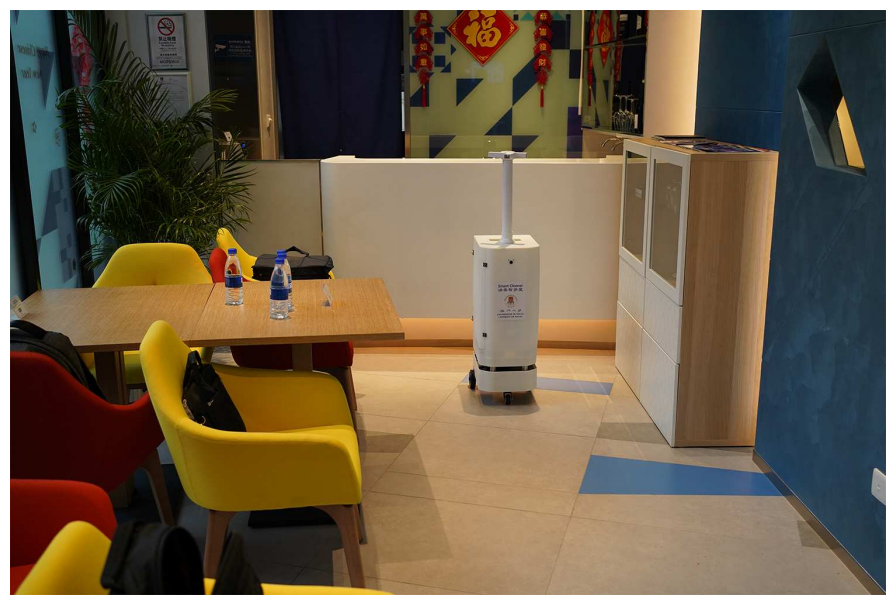

Figure 8. Photo of the developed disinfection robot and the disinfection scene in a hotel cafeteria.

\subsection{Experimental Setup}

The disinfection effect is assessed by measuring the air and surface quality before and after the disinfection work of the robot. To acquire accurate data before and after the disinfection, different methods were used to test the colony-forming unit (CFU) of the air and contact surface of high-frequency use.

For the air quality test, bioaerosol sampling with a single-stage cascade impactor (from SKC BioStage, Inc., Eighty Four, PA, USA) was used, as shown in Figure 9a. During the 
process, all of the procedures meet NIOSH Method 0800 indoor bioaerosol sampling requirements. For the contact surface test, the sterile surface swab kit (from SKC BioStage, Inc.) was adopted, as shown in Figure 9b. The combination of these two methods gives an accurate evaluation of the disinfection effect.



(a)

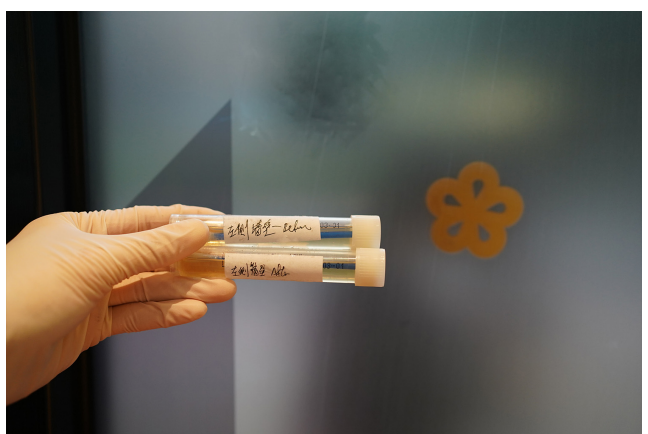

(b)

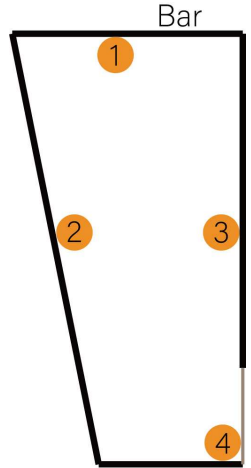

(c)

Figure 9. (a) Air quality testing equipment; (b) object surface quality testing equipment; (c) illustration of the locations of selected sampling points.

For illustration, as shown in Figure 9c, the sampling points for a cafeteria in Holiday Inn Express Hotel Macau were selected as contact surfaces of high-frequency use, including the bar counter, shadow surface under the table, and wall surface.

\subsection{Disinfection Result}

For indoor disinfection, after the environment map establishment, the autonomous disinfection robot patrols according to the preset path to accomplish the disinfection mission of the whole environment. In some cases, like various complicated environments, it can be manually controlled by an operator using a wireless stick controller or keyboard with the assistance of remote vision system to overcome the obstacles.

In this work, the disinfection robot converts hydrogen peroxide liquid into micrometersized drymist particles for an efficient space disinfection by employing the ultrasonic atomizer. Specifically, $6 \%$ hydrogen peroxide liquid (model: Nocolyse, from Oxy'Pharm Ltd., Champigny-sur-Marne, France) is atomized into drymist hydrogen peroxide, which is then diffused into air and surface of the environment. For indoor disinfection, the target density of the drymist hydrogen peroxide is set as $500 \mathrm{ppm}$, which can be detected by using hydrogen peroxide test strips.

The disinfection work is carried out for $20 \mathrm{~min}$ by the developed robot using the drymist hydrogen peroxide. Before and after the disinfection, the testing results for the mentioned hotel cafeteria are tabulated in Table 2 . The testing data are provided by the Institute for the Development and Quality (IDQ), Macau. 
Table 2. Testing results of the air and surface quality before and after disinfection.

\begin{tabular}{lcc}
\hline Specifications & Before & After \\
\hline Total number of air bacteria & $215 \mathrm{CFU} / \mathrm{m}^{3}$ & $35 \mathrm{CFU} / \mathrm{m}^{3}$ \\
\hline Total number of airborne fungi & $232 \mathrm{CFU} / \mathrm{m}^{3}$ & $151 \mathrm{CFU} / \mathrm{m}^{3}$ \\
\hline Total number of bacteria on surface (1) & $1 \mathrm{CFU} / \mathrm{mL}$ & $0 \mathrm{CFU} / \mathrm{mL}$ \\
\hline Total number of bacteria on surface (2) & $1 \mathrm{CFU} / \mathrm{mL}$ & $0 \mathrm{CFU} / \mathrm{mL}$ \\
\hline Total number of bacteria on surface (3) & $1 \mathrm{CFU} / \mathrm{mL}$ & $0 \mathrm{CFU} / \mathrm{mL}$ \\
\hline Total number of bacteria on surface (4) & $1 \mathrm{CFU} / \mathrm{mL}$ & $0 \mathrm{CFU} / \mathrm{mL}$ \\
\hline
\end{tabular}

From Table 2, it can be observed that the disinfection robot has reduced the total numbers of air bacteria and airborne fungi by $83.7 \%$ and $34.9 \%$, respectively. This indicates that the air quality has been significantly improved by the disinfection work. The reason why the air bacteria and airborne fungi are not totally cleaned lies in the fact that the indoor space is open and not closed during the disinfection work. As a result, undisinfected air flows into the working space from time to time during the disinfection process.

Moreover, after disinfection, the total numbers of bacteria for the four sampled surfaces are all reduced to zero. The results reveal that the surfaces of the disinfected space have been thoroughly cleaned by the disinfection robot. The results of the indoor space disinfection demonstrate the great potential of the developed disinfection robot to substitute humans to conduct disinfection work in dangerous contaminated environments.

Even though there are several commercial auto-disinfection robots on the market, their prices are too high to be widely applied for indoor disinfection. To extensively disinfect and sterilize the community environments, it is desirable to develop low-cost and effective autonomous robots for wide application in automated disinfection. The work presented in this paper provides such a cost-effective solution. Based on the state-of-the-art technologies involving navigation, obstacle avoidance, disinfection, and so on, the developed autonomous disinfection robot system is suitable for extensive disinfection applications.

\section{Conclusions}

In this paper, we present the design and verification of a cost-effective autonomous mobile disinfection robot for indoor disinfection based on SLAM and ROS. The robot was developed to conduct deep disinfection work for indoor air and surface by using the approach of drymist hydrogen peroxide. A prototype robot system called Smart Cleaner has been fabricated by integrating the functions of navigation, path planning, and drymist hydrogen peroxide disinfection using ROS programming. The developed robot system has been deployed and tested in real indoor environments, including laboratory, library, hospital, hotel, etc. The results of the disinfection test show that the developed disinfection robot exhibits great effectiveness in combating the COVID-19 pandemic. In future work, a more powerful atomizer will be integrated into the robot to reduce the disinfection time. In addition, the robot vision provided by RGB-D camera will be fused with the 2D-lidar toward an integrated navigation.

Author Contributions: Q.X. proposed the concept design idea; K.R. and Z.W. performed the literature view and mechanism design; K.R. and Z.W. conducted the prototype fabrication and experimental study; K.R., Z.W. and Q.X. wrote the paper. All authors have read and agreed to the published version of the manuscript.

Funding: This research was funded in part by The Science and Technology Development Fund, Macau SAR (File no. 0008/2020/A, 0153/2019/A3, and 0022/2019/AKP) and University of Macau (File no. MYRG2018-00034-FST and MYRG2019-00133-FST).

Institutional Review Board Statement: Not applicable.

Informed Consent Statement: Not applicable. 
Conflicts of Interest: The authors declare no conflict of interest. The founding sponsors had no role in the design of the study; in the collection, analyses, or interpretation of data; in the writing of the manuscript and the decision to publish the results.

\section{References}

1. WHO. WHO Coronavirus Disease (COVID-19) Dashboard. Website. 2021. Available online: https://covid19.who.int/ (accessed on 4 March 2021).

2. Holland, J.; Kingston, L.; McCarthy, C.; Armstrong, E.; O’Dwyer, P.; Merz, F.; McConnell, M. Service Robots in the Healthcare Sector. Robotics 2021, 10, 47. [CrossRef]

3. Yang, G.Z.; Nelson, B.J.; Murphy, R.R.; Choset, H.; Christensen, H.; Collins, S.H.; Dario, P.; Goldberg, K.; Ikuta, K.; Jacobstein, N.; et al. Combating COVID-19-The role of robotics in managing public health and infectious diseases. Sci. Robot. 2020, 5, eabb5589. [CrossRef]

4. Bogue, R. Robots in a contagious world. Ind. Robot 2020, 47, 642-673. [CrossRef]

5. Wang, X.V.; Wang, L. A literature survey of the robotic technologies during the COVID-19 pandemic. J. Manuf. Syst. 2021. [CrossRef]

6. Shen, Y.; Guo, D.; Long, F.; Mateos, L.A.; Ding, H.; Xiu, Z.; Hellman, R.B.; King, A.; Chen, S.; Zhang, C.; et al. Robots under COVID-19 Pandemic: A Comprehensive Survey. IEEE Access 2020, 9, 1590-1615. [CrossRef]

7. Zeng, Z.; Chen, P.J.; Lew, A.A. From high-touch to high-tech: COVID-19 drives robotics adoption. Tour. Geogr. 2020, 22, 724-734. [CrossRef]

8. Li, S.Q.; Guo, W.L.; Liu, H.; Wang, T.; Zhou, Y.Y.; Yu, T.; Wang, C.Y.; Yang, Y.M.; Zhong, N.S.; Zhang, N.F.; et al. Clinical application of an intelligent oropharyngeal swab robot: Implication for the COVID-19 pandemic. Eur. Respir. J. 2020, 56, 2001912. [CrossRef]

9. Medicaroid Corporation, Developing an Automated PCR Viral Testing Robot System. Website. 2021. Available online: https://global.kawasaki.com/en/corp/sustainability/covid19/pcr.html (accessed on 6 June 2021).

10. Liu, T.; Liao, Q.; Gan, L.; Ma, F.; Cheng, J.; Xie, X.; Wang, Z.; Chen, Y.; Zhu, Y.; Zhang, S.; et al. Hercules: An Autonomous Logistic Vehicle for Contact-Less Goods Transportation During the COVID-19 Outbreak. IEEE Robot. Autom. Mag. 2021, 28 , 48-58. [CrossRef]

11. Keenon Robotics Co., L. Keenon Food Delivery Robot Enters Wuhan Fangcai Hospital to Deliver Materials and Food. Website. 2020. Available online: http:/ / www.jjrmyy.com/menzhenzhuanjia/390692.html (accessed on 6 June 2021).

12. Weber, D.J.; Rutala, W.A.; Anderson, D.J.; Chen, L.F.; Sickbert-Bennett, E.E.; Boyce, J.M. Effectiveness of ultraviolet devices and hydrogen peroxide systems for terminal room decontamination: Focus on clinical trials. Am. J. Infect. Control 2016, 44, e77-e84. [CrossRef] [PubMed]

13. Lu, Y.; Hu, G.Q.; Li, Y.; Cai, R.; Lu, L.X.; Yu, X.Z. Comparison study on germicidal effeicacy of two types of hybrogen peroxide system in the wards. Chin. J. Disinfect. 2019, 36, 92-98.

14. Fu, L.; Liu, W. Comparison study on germicidal effeicacy of two types of hybrogen peroxide system in the wards. China Pharm. 2017, 20, 340-344.

15. McGinn, C.; Scott, R.; Donnelly, N.; Roberts, K.L.; Bogue, M.; Kiernan, C.; Beckett, M. Exploring the Applicability of RobotAssisted UV Disinfection in Radiology. Front. Robot. AI 2021, 7, 193. [CrossRef]

16. Freyssenet, C.; Karlen, S. Plasma-activated aerosolized hydrogen peroxide (aHP) in surface inactivation procedures. Appl. Biosaf. 2019, 24, 10-19. [CrossRef]

17. Rudnick, S.N.; McDevitt, J.J.; First, M.W.; Spengler, J.D. Inactivating influenza viruses on surfaces using hydrogen peroxide or triethylene glycol at low vapor concentrations. Am. J. Infect. Control 2009, 37, 813-819. [CrossRef]

18. Goyal, S.M.; Chander, Y.; Yezli, S.; Otter, J.A. Evaluating the virucidal efficacy of hydrogen peroxide vapour. J. Hosp. Infect. 2014, 86, 255-259. [CrossRef]

19. Bailey, T.; Durrant-Whyte, H. Simultaneous localization and mapping (SLAM): Part II. IEEE Robot. Autom. Mag. 2006, 13, 108-117. [CrossRef]

20. Liu, T.; Liu, H.; Li, Y.; Zhang, Z.; Liu, S. Efficient Blind Signal Reconstruction With Wavelet Transforms Regularization for Educational Robot Infrared Vision Sensing. IEEE/ASME Trans. Mechatronics 2019, 24, 384-394. [CrossRef]

21. Barber, R.; Crespo, J.; Gomez, C.; Hernamdez, A.C.; Galli, M. Applications of Mobile Robots; Chapter Mobile Robot Navigation in Indoor Environments: Geometric, Topological, and Semantic Navigation; IntechOpen: London, UK, 2018

22. Chatterjee, A.; Rakshit, A.; Singh, N.N. Vision Based Autonomous Robot Navigation: Algorithms and Implementations; Springer: Berlin, Germany, 201

23. Minguez, J.; Lamiraux, F.; Laumond, J.P. Springer Handbook of Robotics; Chapter Motion Planning and Obstacle Avoidance; Springer: Berlin, Germany, 2008; pp. 827-852.

24. Pandey, A.; Pandey, S.; Parhi, D.R. Mobile robot navigation and obstacle avoidance techniques: A review. Int. Robot. Autom. J. 2017, 2, 96-105. [CrossRef]

25. Gu, J.; Zhou, K.; Liang, T. Numerical Simulation of Vaporous Hydrogen Peroxide Flow Field for Space Disinfection. In Proceedings of the 2019 International Conference on Intelligent Computing, Automation and Systems (ICICAS), Chongqing, China, 6-8 December 2019; pp. 189-191. [CrossRef] 
26. Kampf, G.; Todt, D.; Pfaender, S.; Steinmann, E. Persistence of coronaviruses on inanimate surfaces and their inactivation with biocidal agents. J. Hosp. Infect. 2020, 104, 246-251. [CrossRef] [PubMed]

27. Centers for Disease Control and Prevention. Guideline for Disinfection and Sterilization in Healthcare Facilities. Website. 2008. Available online: https://www.cdc.gov/infectioncontrol/guidelines/disinfection/disinfection-methods/chemical.html (accessed on 6 June 2021).

28. National Health Commission of China. Notice of the General Office of the National Health Commission on Issuing Guidelines for the Use of Disinfectants. Website. 2020. Available online: http://www.nhc.gov.cn/zhjcj/s9141/202002/b9891e8c86d141a0 8ec45c6a18e21dc2.shtml (accessed on 6 June 2021).

29. Gul, F.; Rahiman, W.; Alhady, S.S.N. A comprehensive study for robot navigation techniques. Cogent Eng. 2019, 6, 1632046. [CrossRef]

30. Patle, B.K.; L, G.B.; Pandey, A.; Parhi, D.R.K.; Jagadeesh, A. A review: On path planning strategies for navigation of mobile robot. Cogent Eng. 2019, 15, 582-606. [CrossRef]

31. Wen, S.; Zhao, Y.; Yuan, X.; Wang, Z.; Zhang, D.; Manfredi, L. Path planning for active SLAM based on deep reinforcement learning under unknown environments. Intell. Serv. Robot. 2020, 13, 263-272. [CrossRef]

32. Li, C.; Zhang, X.; Gao, H.; Wang, R.; Fang, Y. Bridging the Gap Between Visual Servoing and Visual SLAM: A Novel Integrated Interactive Framework. IEEE Trans. Autom. Sci. Eng. 2021. [CrossRef]

33. Wang, C.; Meng, L.; She, S.; Mitchell, I.M.; Li, T.; Tung, F.; Wan, W.; Meng, M.Q.H.; de Silva, C.W. Autonomous mobile robot navigation in uneven and unstructured indoor environments. In Proceedings of the 2017 IEEE/RSJ International Conference on Intelligent Robots and Systems (IROS), Vancouver, BC, Canada, 24-28 September 2017; pp. 109-116.

34. SLAMTEC. The Rplidar ROS Package, Support Rplidar A2/A1 and A3/S1. Website. 2021. Available online: http://wiki.ros.org/ rplidar (accessed on 6 June 2021).

35. Gerkey, B. This Package Contains a ROS Wrapper for OpenSlam's Gmapping. Website. 2021. Available online: http: / / wiki.ros.org/gmapping (accessed on 6 June 2021).

36. Marder-Eppstein, E. Movebase. Website. 2021. Available online: http:/ /wiki.ros.org/movebase (accessed on 6 June 2021).

37. Washio, T.S. OPENNI2. Website. 2021. Available online: https://github.com/OpenNI/OpenNI2 (accessed on 6 June 2021).

38. Gerkey, B.P. AMCL Is a Probabilistic Localization System for a Robot Moving in 2D. Website. 2021. Available online: http:/ / wiki.ros.org/amcl (accessed on 6 June 2021).

39. Ferguson, M. Rosserial. Website. 2021. Available online: http:/ / wiki.ros.org/rosserial (accessed on 6 June 2021).

40. Grisetti, G.; Stachniss, C.; Burgard, W. Improved Techniques for Grid Mapping With Rao-Blackwellized Particle Filters. IEEE Trans. Robot. 2007, 23, 34-46. [CrossRef]

41. Hart, P.E.; Nilsson, N.J.; Raphael, B. A Formal Basis for the Heuristic Determination of Minimum Cost Paths. IEEE Trans. Syst. Sci. Cybern. 1968, 4, 100-107. [CrossRef]

42. Seder, M.; Petrovic, I. Dynamic window based approach to mobile robot motion control in the presence of moving obstacles. In Proceedings of the 2007 IEEE International Conference on Robotics and Automation (ICRA), Roma, Italy, 10-14 April 2007; pp. 1986-1991. [CrossRef]

43. Shapiro, S.S.; Wilk, M.B. An analysis of variance test for normality (complete samples). Biometrika 1965, 52, 591-611. [CrossRef] 